\title{
The influence of attention distraction on the drivers' behaviour
}

\author{
Kamila Gąsiorek ${ }^{1, *}$, Adam Tarnowski ${ }^{1}$, and Justyna Harasimczuk ${ }^{2}$ \\ ${ }^{1}$ Motor Transport Institute, Transport Management and Telematics Centre, Warsaw, Poland \\ ${ }^{2}$ Cardinal Stefan Wyszyński University, Institute of Psychology, Warsaw, Poland
}

\begin{abstract}
Distraction of the driver's attention is one of the key aspects of research conducted by automotive companies. It concerns not only onboard devices, but also the factors outside the vehicle, such as advertising displays. Too much attention focused on advertisements can have negative impact on the driver's behaviour and driving quality. The 2-second distraction can more than double the risk of an accident. The paper presents the results of a simulator-based study, in which 60 drivers (including 30 women) age- 18-64 take part. All participants had a valid driving license and differed in their driving experience. It has been verified how the time of focusing the attention on the roadside advertisements affects the given safety and driving performance indicators.
\end{abstract}

\section{Introduction}

External factors, including advertisements, cause about $10 \%$ of traffic collisions [1]. At the same time, it is estimated that the number of eye fixations and their duration in the presence of the roadside adverts along the non-urban roads is from 5 to $10 \%$ higher than in the absence of these advertisements [2]. This directly translates into decreasing the concentration level of the driver and extending his or her potential reaction time [3]. In the presence of the roadside advertisements, drivers have problems with staying in the lane. This is manifested by the lengthened periods of the adjacent road lane encroachment, changes in the speed and involuntary reduction of the distance to the preceding vehicle [4]. Such behaviours are associated with an increased likelihood of dangerous road traffic incidents. Existing research suggests that any interference causing driver's eyes to turn away from the road for more than 2 seconds significantly increases the risk of an accident $[5,6]$.

In Herrstedt and Greibe's study [4] it is estimated that $44 \%$ of all glances (fixations) directed at the roadside adverts last for $0.5 \mathrm{~s}$ or longer. During this time a driver moving with an allowed speed of $90 \mathrm{~km} / \mathrm{h}$ will travel at least $12.5 \mathrm{~m}$. In order to objectively present the threats resulting from the observed finding, the term "safety buffer" was defined. It is the time in which the driver has to respond to the sudden traffic situation. Its value equals the difference between the time to collision with the preceding vehicle and the time of eye fixation on the advert when driving behind the vehicle. The analyses have shown that $1 / 4$ of

\footnotetext{
*Corresponding author: kamila.gasiorek@,its.waw.pl
} 
the glances at the adverts was associated with a simultaneous reduction of the time buffer below the safety limit of $2 \mathrm{~s}$, and for $1 / 5$ of such glances the time buffer was $1.5 \mathrm{~s}$ and less $[5]$.

The above-mentioned indicators of risky behaviours are associated with the phenomenon of excessive cognitive load. It increases along with the requirements of the road situation, leading to task interference. The driver observes and analyses the content of the advertisement instead of focusing on the primary task - the driving. This interference is particularly dangerous for inexperienced drivers who are prone to distraction. These indicators may be higher on sections with high accumulation of the roadside advertising $[2]$.

\section{Current Study}

The test was carried out to verify the 2-second rule in the Area of Interest, according to which the driver should not take his eyes off the road for more than 2 seconds. If this time is longer, the driver may become distracted [6-8]. A wide range of vehicle motion and control parameters were taken into account to identify the ones making the greatest impact on distraction. During the experiment, the parameters of vehicle steering were recorded, such as steering wheel angle, time headway or time to collision, as well as vehicle motion parameters, for example speed or position in the lane. The study was divided into two parts. Each participant took a drive with the driving simulator twice.

\subsection{Description of the research experiment}

The research experiment was based on three types of tasks. Two of them were treated as basic tasks and were connected with driving a vehicle. We used the tasks well described in the literature and frequently used in simulator-based studies: Three Vehicle Platoon Task (3VPT) and Lane Change Task (LCT) $[9,10]$. The 3VPT scenario is based on driving a vehicle in a convoy. It requires the driver to control lateral position, longitudinal position, as well as to detect and respond to the programmed stimuli. The driver's task was to drive as the central vehicle in a column consisting of three cars. The participant was supposed to maintain an appropriate distance from the preceding vehicle, approximately 35 meters. The preceding vehicle was moving at a constant speed of $80 \mathrm{~km} / \mathrm{h}$ and did not perform any manoeuvres. The driver drove along a straight three-lane carriageway. The ambient conditions, i.e. weather and lighting were controlled during the experiment.

The second scenario was based on the Lane Change Task (LCT). It consisted of driving along a straight road at a constant speed and required the driver to constantly change the position between the three lanes, according to the information presented on 24 road signs. The signs were appearing at regular intervals to the right of the track.

The additional task performed both during the 3VPT and the LCT task was to observe advertising displays located near the road. All displays had the same content: red dots on a white background. The drivers had to focus their attention on the dots until the advertisement disappeared from the field of view. In two conditions the dots were present for either 1 second or 2 seconds. In the control group, no advertising was displayed. 

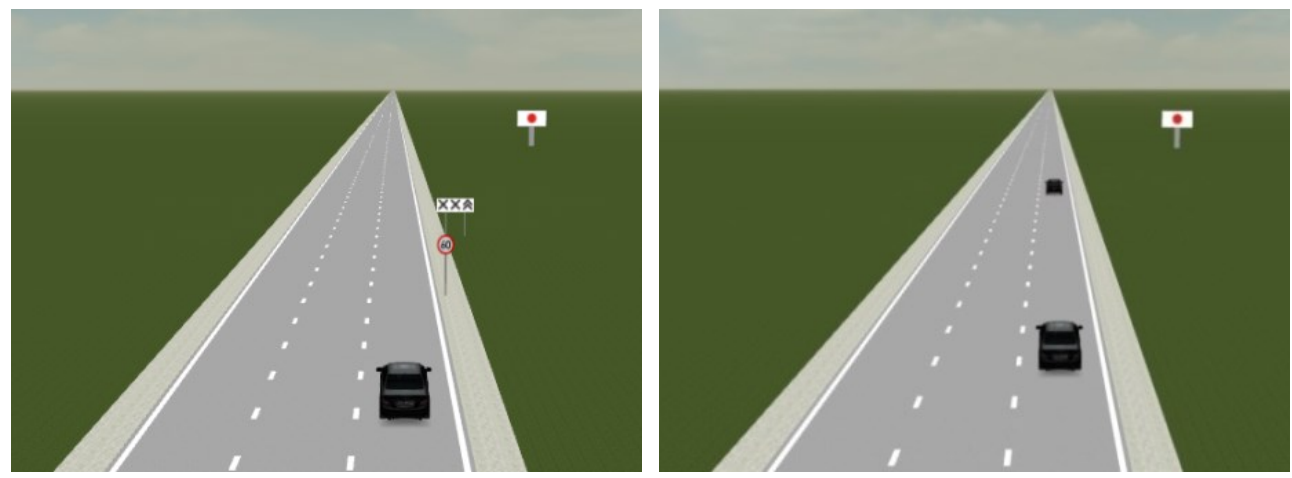

Fig. 1. Screenshots from the scenarios for both tasks. Source: own elaboration.

\subsubsection{Participants}

The study was conducted on a group of 60 drivers, age 18-64 (50\% women). The participants were divided into three age groups: under 25 years, $30-40$ years, $55-65$ years of age and with respect to the driving experience. Inexperienced drivers were characterized by having their driving licenses for a maximum of three years and by declaring to drive only occasionally. Experienced drivers had to have a driving license for more than 3 years and drive around 9,000 km during this period. Participants were recruited for the study by telephone interviewing through an external research company. They received remuneration for participating in the study.

\subsubsection{Procedure}

The research experiment was carried out in accordance with the research methodology developed for the use of car driving simulators, at the Motor Transport Institute. Each participant first performed an adaptive 10-minute drive, during which he had the opportunity to get used to controlling the vehicle. Adaptation was also aimed at eliminating people particularly sensitive and feeling nauseous while driving in a simulator. During the adaptation drive, participants had to drive through the highway, the local built-up area road and the non-urban road. Then, their task was to drive behind the vehicle and maintain a distance of about $35 \mathrm{~m}$ from it. The current distance from the preceding vehicle was displayed on the screen. After the adaptation drive, the participants had a 10-minute break. After that they had to complete two test scenarios: first the 3VPT task, and then the LCT task. Between the two scenarios they had a 5-minute break. Participants from each age and sex group were divided into three subgroups according to the experimental manipulation conditions. The first subgroup watched the displayed dots for 1 second, the other for 2 seconds, and the third had no advertising displays to watch.

\subsubsection{Method}

The experiment was carried out using a high-end AutoSim AS 1200-6 simulator. It is built from a full-size cabin of Opel Astra IV passenger car and equipped with a system of projectors displaying the image on a screen covering $200^{\circ}$ of visual field and a system of screens that fulfil the function of car mirrors. The cabin is mounted on the 6 DOF (degrees of freedom) platform, allowing to simulate angular and linear movements of the cabin during actual driving. 


\subsection{Results}

\subsubsection{The impact of completing additional tasks on the driving parameters in the 3VPT scenario}

In order to verify the impact of the time of the driver's vision reversal from the road on the vehicle driving parameters, the statistical analyses were carried out. They showed significant indicators of the changes depending on the length of the advertising displays observations. For the 3VPT task the parameters with significant differences were: the vehicle's speed, the acceleration pedal pressure and changes in the steering wheel angle. The analysis was made for two time periods. First, we examined the impact of the additional task on the time during the exposure of the stimulus (carriers with dots), and then for 2 seconds after the appearance of the stimulus. For the LCT task, parameters determining the position in the cross-section of the lane were determined: reference field and ID field. Changes in these parameters are described below (subsection 2.2.2).

The time when the drivers took their eyes off the road had a statistically significant impact on the vehicle's speed, especially regarding its reduction $(F(2,57)=4.508$; $\mathrm{p}=0.015$ ). The difference was found for the group with a condition of $2 \mathrm{~s}$ stimulus exposure time and a control group $(\mathrm{p}=0.022)$. The results show that the drivers' focus on the additional task (observation of roadside advertisement) required reducing the difficulty of the basic task, which resulted in a change (reduction) in the driving speed. The effect was particularly evident among younger drivers. The results are presented in Figure 2.

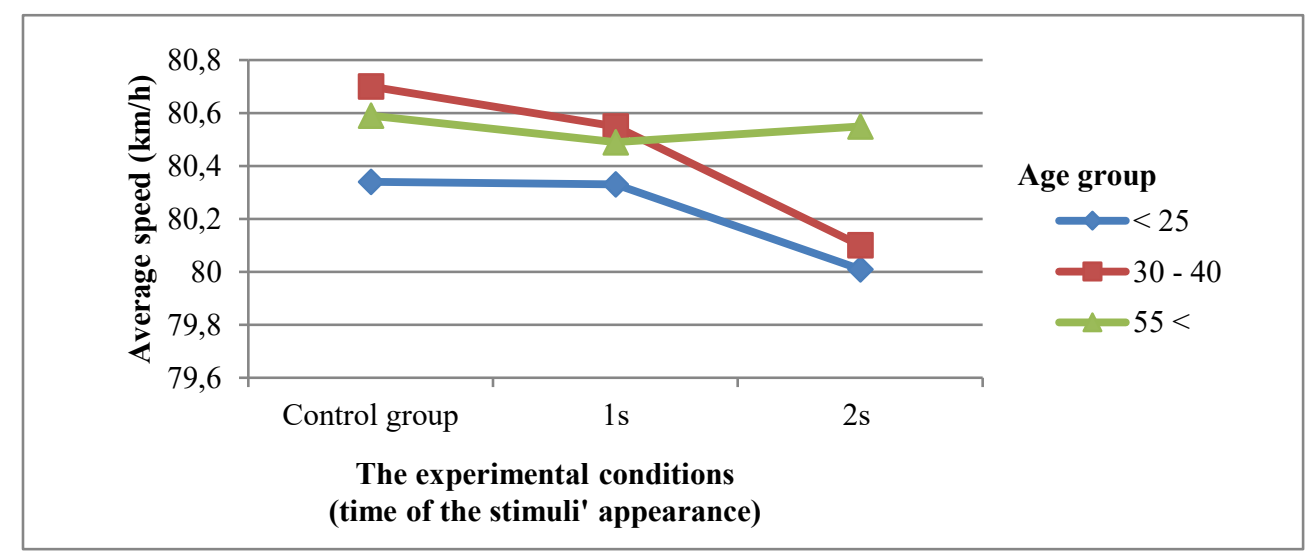

Fig. 2. The average vehicle speed while performing the additional task (observation of advertising display for a specified period of time in the experimental groups and the control group). Source: own elaboration.

The analysis conducted for 2 seconds period after the stimulus appeared showed similar results. The longer the driver turns his eyes away from the traffic situation, the more he/she reduces the speed. However, this was only a statistical trend.

In the 3VPT task the degree of pressing the accelerator pedal was also indicated as a parameter changing along with the observation time of the advertising display $(F(2,57)=$ $3.591 ; \mathrm{p}=0.034)$. The analyses conducted of the interactions during carrier exposure revealed significant differences between the group with the stimulus appearance time of $2 \mathrm{~s}$ and the control group. During a larger (2-second) distraction, the drivers significantly reduced the pressure of the accelerator pedal compared to drivers who did not see the advertising displays while driving $(\mathrm{p}=0.036)$. The impact was especially evident in the group of less experienced drivers under the age of 25 and a group of participants in the 
middle age with more experience compared to the group of the oldest drivers. In the group of people over 55 years old, the pressure of the accelerator pedal was significantly affected by a distraction of the driver's attention lasting one second. The results are shown in Figure 3.

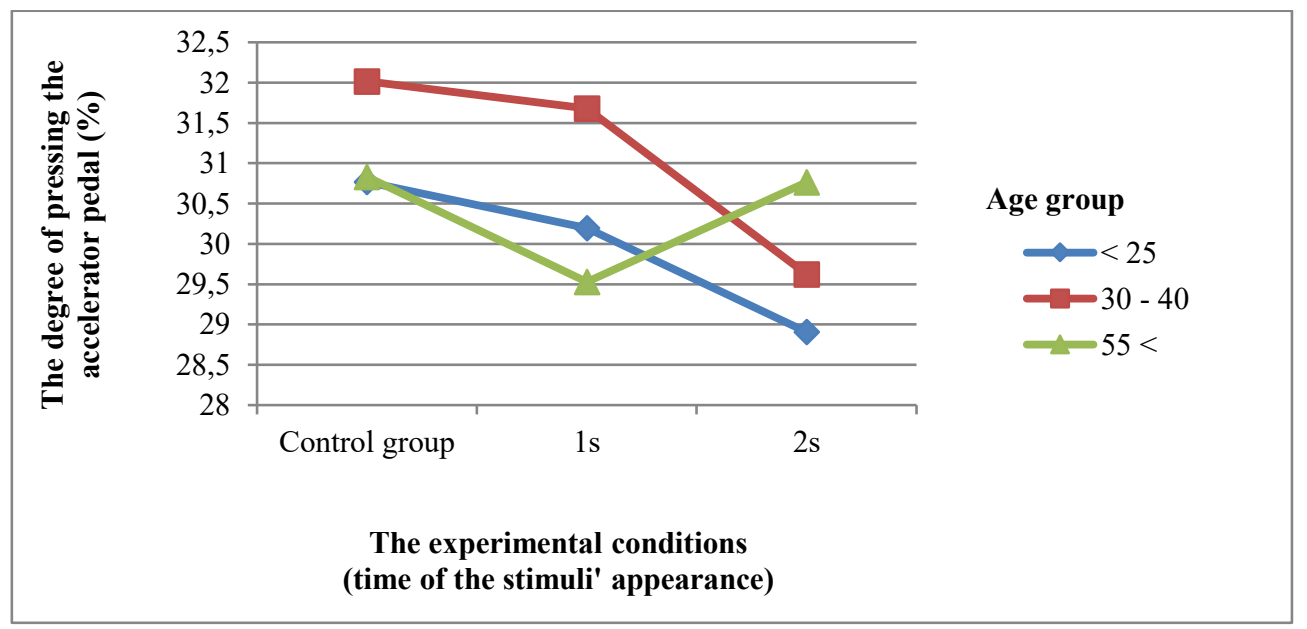

Fig. 3. The rate of the pressure of the accelerator pedal while performing an additional task (observation of advertising displays for a specified period of time in the experimental groups and the control group). Source: own elaboration.

During the 3VPT task, the time the driver's eyesight was off the road also had an effect on changes in the angle of the steering wheel. The result was recorded at the level of tendencies. The analyses indicate the decrease in the driving performance. The highest indicator of the average position of the driver's wheel was noted in the group of participants exposed to the advertising displays for 2 seconds, which indicates that these drivers had the greatest difficulty in maintaining the position of the steering wheel.

\subsubsection{The effect of completing additional task on the driving parameters in the LCT task}

Analyses of the data gathered in the LCT task concerned changes in the position in the cross-section of the lane. This position indicates the location of the fixed point of the vehicle on the horizontal axis in relation to the determined cross-sectional point of the lane. When assessing the quality of the performed driving task, this parameter is considered as an indicator of the driver's ability to control the vehicle's movement.

To measure this indicator in the experiment, the reference points for the vehicle and lane were set. Two variables were assumed as the reference points of the road. First, the geometric centre of the lane for the 'ID field' parameter and, second, the average position of the vehicle in the lane (characteristic for each driver) with stabilized driving straight ahead for the 'reference field' parameter. The vehicle's reference point was located in the centre of the rear axle of the vehicle. Based on the variables, we estimated the 'reference field' (field under the driving curve in relation to the reference curve) and the 'ID field' (field under the driving curve in relation to the curve determined by the centres of the lanes). We analysed fields for 18 signs informing about lane change. The size of the field is a measure of the inaccuracy of the manoeuvre.

Obtained results for both 'reference fields' and 'ID fields' indicate a deterioration in the driving performance during the exposure of advertising displays lasting 1 second. The 
finding may indicate that the limits of cognitive abilities of drivers in this research group have been exceeded. In the group of drivers exposed to advertising for 2 seconds, a significant decrease in parameter values was recorded in comparison to the 1 -second exposure group and the control group. The significant relationships found for the 4 'reference fields' and 4 'ID fields' are shown in Fig. 6. and Fig. 7.

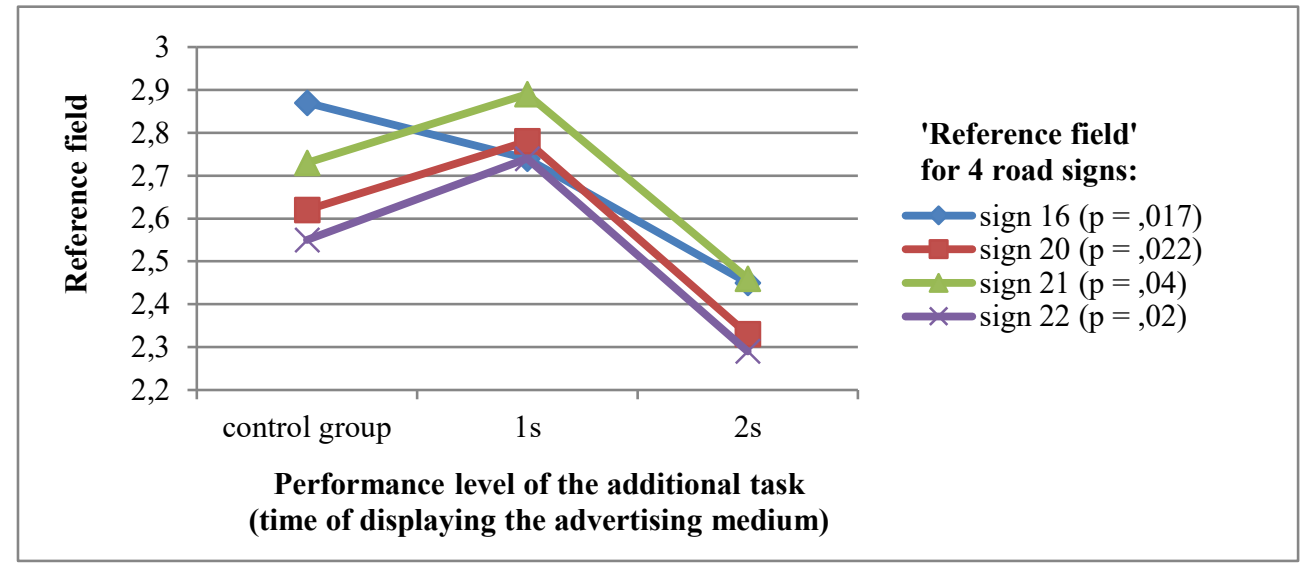

Fig. 5. Graph of the 'reference field' for 4 road signs, measured while performing an additional task (the observation of advertising displays for a specified period of time). Source: own elaboration.

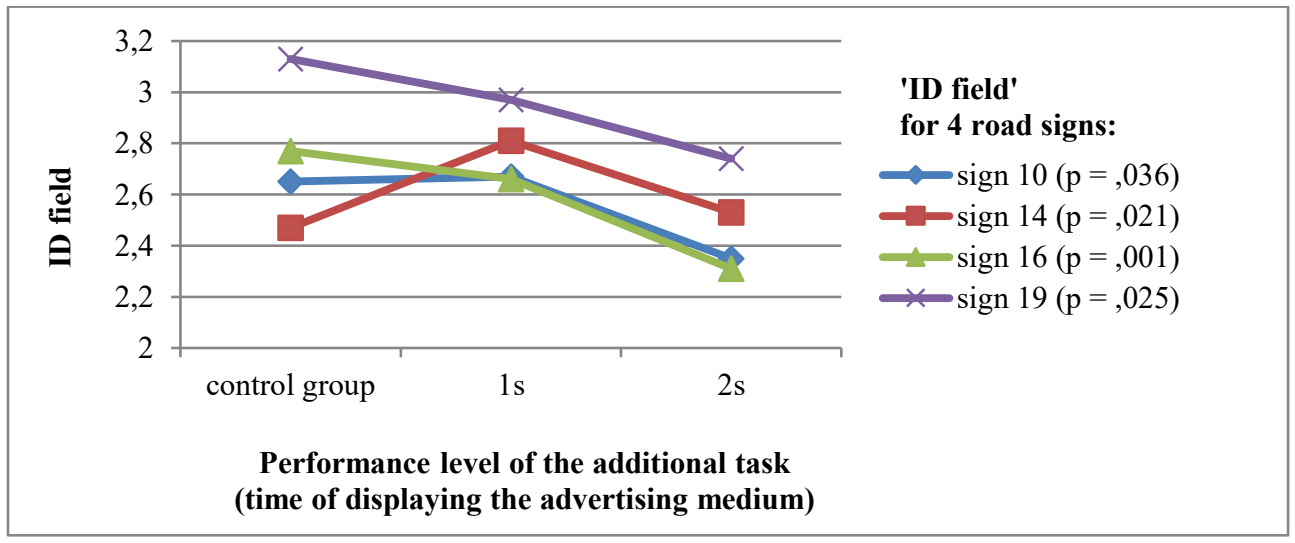

Fig. 6. Graph of the 'ID field' for 4 road signs, measured while performing an additional task (the observation of advertising displays for a specified period of time). Source: own elaboration.

Similar patterns of the results were found for the analysis of the mean 'reference field' and the mean 'ID field', which are mean field size indicators for all cases of a given type of manoeuvre. The analyses revealed significant differences between the group of drivers who were exposed to the ads for 1 second and the other two groups- 2 -seconds group $(p=0.029)$ and control group $(\mathrm{p}=0.015)$, during the lane change manoeuvre from the left to the central lane. We assume that drivers in the 1-second group were having more difficulties to control the vehicle when performing this particular manoeuvre.

\section{Conclusions}

The conducted experiment indicates that roadside advertising displays may disturb the driver's ability to perceive and understand safety-relevant information and have an impact 
on the control of the vehicle. This is suggested by the parameters of driving performance, changing accordingly to the time of displaying the advertising. These parameters are: speed, accelerator pedal pressure intensity and the steering wheel angle. We assume that the drivers change their behaviour in order to reduce the task load. However, the results do not confirm the hypothesis enough, that taking eyes off the road for 2 seconds has a significant impact on the driver's distraction. We did not observe any significant difference in the distance from the preceding vehicle or the time to collision.

The results obtained in the Lane Change Task scenario (LCT) may suggest that the change rate in the drivers' visual field is more distracting than taking eyes off the road. Distraction for 1 second turned out to have a greater negative impact on the driving performance than looking away from the road situation for a longer time. This phenomenon is associated with the processes of rapid eye movements and can be explained using the model proposed by Findlay-Walker [11]. During the eye fixation visual system simultaneously performs the cognitive analysis of the object, located in the central visual field and selects the next fixation point, which has the highest priority. The processes that attract the eye to the new location provides the map of priorities, which is determined on the basis of the task relevance assessment of individual elements, including the initial, peripheral cognitive analysis of the object in the new location. The decision to move the eye depends on the above-mentioned processes and is undertaken when the cognitive analysis at the fixation point is completed. Then, the priority on another point becomes stronger. We assume that the cognitive analysis of the advertising displayed for 1 second in our experiment was not brought by drivers to a satisfactory state, thus the decisions to move the eye were being forced and took place too quickly. The disruption of the eye movements cycle could reduce the efficiency of cognitive processing and, as a result, contribute to reducing the driver's ability to control the vehicle. These conclusions should be the subject of further research, but they constitute an important signal that drivers' distraction phenomenon is more complex than it initially seemed and does not always depend linearly on the simple characteristics of the exposed stimuli.

\section{References}

1. M.S. Young, J.M. Mahfoud, Driven to distraction: The effects of roadside advertising on driver attention, Contemp. Ergon. 44 pp. 145-150 (2007)

2. M.S. Young, J.M. Mahfoud, N.A. Stanton, P.M. Salmon, D.P. Jenkins, G.H. Walker, Conflicts of interest: The implications of roadside advertising for driver attention, Transp. Res. Part F Traffic Psychol. Behav. 12 pp. 381-388 (2009). doi:10.1016/j.trf.2009.05.004

3. H. Marciano, Y. Yeshurun, Perceptual load in central and peripheral regions and its effects on driving performance: Advertising billboards, Work. 41 pp. 3181-3188 (2012). doi:10.3233/WOR-2012-0580-3181

4. L. Herrstedt, P. Greibe, P. Andersson, Roadside Advertising Affects Driver Attention and Road Safety, in: Proc. 3rd Int. Conf. Driv. Distraction Ina. Sept. 4-6, 2013, (No. 05-P), pp. 1-14 (2013)

5. S.G. Klauer, T. a. Dingus, V.L. Neale, J.D. Sudweeks, D.J. Ramsey, The Impact of Driver Inattention on Near-Crash/Crash Risk: An Analysis using the 100-car Naturalistic Driving Study Data. Administration Report No. DOT HS 810594 (National Highway Traffic Safety Administration, Washington, D.C., 2006)

6. T.H. Rockwell, Spare visual capacity. New empirical results for an old idea, Vis. Veh. -- II. pp. 317-324 (1988) 
7. AAM Driver Focus-Telematics Working Group, Statement of Principles, Criteria and Verification Procedures on Driver Interactions with Advanced In- Vehicle Information and Communication Systems Driver Focus-Telematics Working Group. Available at: http://www.autoalliance.org/index.cfm?objectid=D6819 (2006)

8. Visual-manual NHTSA driver distraction guidelines for in-vehicle electronic devices. Available at: https://www.federalregister.gov/articles/2012/02/24/2012-4017/visualmanual-nhtsa-driver-distraction-guidelines-for-in-vehicle-electronic-devices (National Highway Traffic Safety Administration, Washington DC, 2012)

9. M. Kruszewski, M. Niezgoda, T. Kamiński, M. Nader, Pilot study over secondary task cognitive workload induced on drivers in AS 1200-6 simulator, Logist. Transp. 36 pp. 69-78 (2017)

10. T. Petzoldt, N. Bär, C. Ihle, J.F. Krems, Learning effects in the lane change task (LCT) -Evidence from two experimental studies, Transp. Res. Part F Traffic Psychol. Behav. 14 pp. 1-12 (2011). doi:10.1016/j.trf.2010.09.001

11. A. Tarnowski, Pamięć robocza i mechanizmy kontroli ruchów oka, 300 milisekund $z$ życia człowieka (Wydawnictwo Instytutu Psychologii PAN, 2009) 